\title{
Opinión
}

\section{Tabacosis, un cambio de terminología para un aumento de conciencia}

\author{
(Tabacosis, a change in therminology for an increase in consciousness)
}

Alexis Rodríguez-Madrigal ${ }^{1}$, Miguel Angel 0 guilve-Méndez²

\section{Resumen}

Ante la ausencia de definición categórica de la Real Academia Española, tenemos que la Enfermedad Pulmonar Obstructiva Crónica (EPOC) es prevenible y tratable, con afectación sistémica extrapulmonar, donde el componente pulmonar se caracteriza por limitación al flujo del aire que no es completamente reversible y es progresiva. ${ }^{1}$ Esta es probablemente una de las definiciones médicas con mayor cantidad de posiciones e intereses encontrados y es que, indudable y lastimosamente, las siglas EPOC, fuera del gremio médico, no tienen mayor significancia, pues diariamente se encuentra gran cantidad de pacientes portadores de esta patología, que no tienen noción real de su padecimiento, el cual con mucho costo es aclarado por el médico tratante, debido a lo engorroso de su significado, variedad de síntomas y múltiples patologías causales. Estas vicisitudes, en cuanto a la patología causal de EPOC, son las que la han convertido en el “cajón” donde se colocan diversas neumopatías que no tienen un origen específico, sin importar si presentan o no obstrucción al flujo aéreo; además, estos inconvenientes sobre terminología, hacen dificultosa la realización de búsquedas bibliográficas, por la poca acogida que ha tenido el término. ${ }^{2}$

\section{Descriptores: EPOC, tabacosis.}

\section{Abstract}

In the absence of categorical definition of the Real Spanish Academy, we have to Lung Disease Obstructive Disease (COPD) is a preventable and treatable, with extrapulmonary systemic disease, where pulmonary component is characterized by flow limitation Air that is not fully reversible and is progressive. ${ }^{1}$ This is probably one of the medical definitions with greater number of positions and

1 Director Médico. Hospital Fernando Escalante Pradilla.

2 Médico General. Hospital Fernando Escalante Pradilla

Correspondencia: Dr. M. 0 guilve Méndez.

Correo electrónico:moguilve@gmail.com

A partado 335-8000 Pérez Zeledón, SJ, Costa Rica

ISSN 0001-6002/2011/53/2/99-101

Acta Médica Costarricense, @2011

Colegio de Médicos y Cirujanos interests and is found which is undoubtedly and unfortunately, COPD stands outside the medical community have no greater significance because find large numbers of patients daily carriers of this disease that have no real notion of suffering, doubt that many costs are clarified by the physician because of the cumbersome of its meaning, variety of symptoms and it causes many diseases. These vicissitudes in terms of causal pathology COPD, are what have become the "box " where placed various lung diseases that have no origin specifically, whether present or not airflow obstruction air, besides these problems of terminology, make it difficult to carry out literature searches by poor reception given to this término.

Keywords: EPOC, tabacosis.

\section{Historia de La EPOC}

Alcuestionar si en laAntigüedad padecieron neumopatías obstructivas crónicas similares a las que hoy afectan a millones de personas, resulta muy difícil responder. Si se toman en cuenta los principales factores (el tabaco y la contaminación ambiental) causantes del incremento de la enfermedad en la segunda mitad del siglo pasado, resulta obvio que no existían en épocas remotas, o, por lo menos, no en las proporciones actuales. Sin embargo, al indagar en la historia de la humanidad, se obtienen datos de cómo en pulmones de momias egipcias, se han aislado partículas de carbón (antracosis), atribuibles a la inhalación del humo de las hogueras de leña en chozas primitivas con pobre ventilación. ${ }^{3}$

No es sino hasta el siglo XVII, y con base en estudios derivados de autopsias, cuando se conocen las entidades que se denominan con el término EPOC. Sin embargo, hasta finales del siglo XVIII, Laennec, médico francés, estableciò la diferencia entre enfisema y bronquitis, instituyendo así las bases de la neumología moderna.

Ya en 1995, Dornhorst señaló que había dos patologías que podrían llevar al paciente a una insuficiencia respiratoria crónica, las cuales fueron: bronquitis crónica, con su característico "blue bloater” (abotagado azul), y el enfisema 
pulmonar, con su "pink puffer" (soplador rosado). En esa misma época de mediados de siglo XX, se volvió la mirada al tabaquismo y se entrelazó de manera inequívoca, en la génesis de la Enfermedad Pulmonar Obstructiva Crónica, convirtiéndose así en la adicción legal más combatida. ${ }^{3}$

\section{Evolución a EPOC}

El concepto EPOC ha ido evolucionando desde su invención. Anteriormente la patologías pulmonares se dividían de acuerdo con distintos criterios clínicos, como la bronquitis crónica, con su periodos de tos productiva; el asma, con su broncoespasmo comúnmente reversible, o la manera categórica en que se relacionaban tabaquistas y enfisematosos.

En la actualidad todas estas patologías cayeron en un cajón denominado EPOC, y es que probablemente haya muchos intereses encontrados tras todo esto. Lo cierto es que los fumadores neumópatas se libraron del ataque social; su denominación ahora es más "light” al mezclarla con otras patologías, lo que sin duda tranquiliza la conciencia de bastantes fumadores, al no encontrar incluido en su diagnóstico clínico, el causal de su precaria situación respiratoria: el fumado.

\section{Tabaquismo y EPOC en Costa Rica}

El EPOC es una enfermedad causada primariamente por el humo del tabaco (fumador activo y pasivo); informa, además, que a nivel mundial hay cerca de 210 millones de personas con EPOC, y la brecha entre hombres y mujeres está disminuyendo, debido al aumento de mujeres fumadoras Además, se menciona el tabaco como partícipe de 13500 defunciones diarias a nivel mundial. ${ }^{4}$

A nivel nacional, la situación es parecida. En el primer quinquenio de esta década, el Registro Nacional de Tumores reportó que el 37,1\% de los tumores malignos se relacionan con el tabaco, para un total de 13544 casos nuevos de cáncer debido a este nocivo hábito, para el presente año se esperan 307 casos nuevos.

Durante 2009, el 5,64\% del presupuesto de la Seguridad Social se gastó en atención de enfermedades asociadas al uso de tabaco Esto corresponde a 58196 millones de colones, un aumento de 10 mil millones con respecto a 2008, lo cual refleja que cada día aumentan los fumadores y sus morbilidades. Asimismo, en 2009, debido a patologías atribuibles al tabaco, se brindaron 1,1 millones de consultas médicas, 176 mil consultas en servicios de Emergencias, 18 mil incapacidades y 9200 estancias hospitalarias, todo producto del fumado. Estos números, traducidos a millones de colones, arrojan estas alarmantes cifras: 23936 millones utilizados en hospitalizaciones, 29095 por consultas, 4811 por servicio urgencias y 354 millones por incapacidades lo que suma, finalmente, unos 58196 millones de colones, por patologías prevenibles en su totalidad. ${ }^{5}$

\section{EPOC, un cambio de terminología para un aumento de} conciencia

Producto de lo leído con anterioridad, se puede obtener dos conclusiones, que son el pilar central de este escrito. Primeramente, la asociación entre el fumado y la EPOC es indiscutible a nivel mundial, y en el país el tabaquismo es una adicción que muchas veces no se considera enfermedad como tal, lo cual es erróneo. Además, su repercusiones en el organismo son innumerables, al afectar prácticamente todos los sistemas corporales, pero con manifestaciones pulmonares muy evidentes, que son el motivo de debate en este momento.

Como segundo punto y central, la definición del término EPOC genera más dudas que aclaraciones y se considera que debe ser cambiado por uno más explicativo, y que incluya el agente etiológico, por lo menos a nivel nacional.

Existen otras patologías bastante conocidas: hepatitis alcohólica, asbestosis, depresión navideña, estrés laboral, las cuales, en su nombre, incluyen su causal y hacen más sencilla su comprensión, tanto por parte del personal de salud como del paciente.

En la palabra EPOC se crea un vacío inmenso que permite encajonar el tabaquismo, al lado de otras patologías con causales muy distintas. La repercusión de esa denominación tiene un importante carácter social, ya que los fumadores no realizan una asociación directa entre fumar un cigarrillo y una enfermedad llamada EPOC, pues no tienen idea del significado de las siglas, y mucho menos sus causas, síntomas y desenlace.

Desde el punto de vista económico y en el caso hipotético de ser dueños de una tabacalera, nos sentiríamos sumamente agradecidos con el desuso de la palabra enfisema y su sustitución por un término más "light”: EPOC, del cual nuestros clientes saben muy poco o nada, El poder está en el conocimiento y, si existe una población fumadora, en su mayoría ignorante de los alcances del fumado, tendrá una mina de oro de por vida, pero, por supuesto, el objetivo de nuestro gremio no es acumular dinero a costa de perder vidas humanas, sino preservar este don.

La propuesta se basa en darle a la enfermedad pulmonar obstructiva crónica y otras patologías del árbol respiratorio producto del fumado, un nombre propio basado en su etiología, para de esta manera lograr conciencia social y, mayoritariamente, de los fumadores, que en su adicción al cigarrillo avanzan a paso acelerado hacia engrosar las listas de morbimortalidad del país.

La propuesta de nombre que más atinado pareció fue el término “TABACOSIS”, Que incluye el tabaco y el sufijo -osis (“que está siendo afectado con”), lo que asegura la relación directa causa-efecto que no dejará dudas en el personal de salud, ni mucho menos en la sociedad fumadora, 
que sería el objetivo fundamental. Es algo muy simple que puede, a largo plazo, producir un gran cambio en los hábitos de esta población, la cual verá cada día más que sus alteraciones respiratorias son consecuencia de la tabacosis.

Se podría entonces definir la tabacosis como un un grupo de enfermedad de la vía aérea, producto del fumado, que incluye enfisema, fibrosis y neoplasias, desde los labios hasta el último alveolo. Se considera que de no adoptarse una medida de este tipo, el fumado, como causa de morbimortalidad, persistirá y aumentará, encubierto tras la compleja sigla, EPOC, y seguiremos siendo cómplices de un engaño público con el que todos nos vemos perjudicados.

\section{Referencias}

1. Estrategia global para el diagnóstico, tratamiento y prevención de la Enfermedad Pulmonar Obstructiva Crónica (GOLD, por sus siglas en inglés), Actualización 2009.

2. Díaz Lobato S, Mayorales Alises S. Un solo nombre para una sola enfermedad: EPOC. Arch Bronconeumol 2003;39:376-7.

3. Sauret Valet J. EPOC: Un viaje a través del tiempo. Barcelona: Healthnet SL. Rubes Editorial SL; 2000.

4. Organización Mundial de la Salud. Centro de Prensa. Nota Descriptiva $\mathrm{N}^{\circ}$ 315. 2009 en http://www.who.int

\section{Comité Editorial Acta Médica Costarricense \\ Lista de revisión para autores \\ Por favor, complete esta lista antes de enviar su manuscrito. \\ $\square$ Carta de presentación \\ $\square$ Tres copias completas del manuscrito con cuadros y figuras, y en formato digital \\ Página de título \\ $\square$ Título \\ $\square$ Título corto, no mayor de 45 caracteres \\ $\square$ Autor(es) y afiliación(es) \\ $\square$ Lugar donde se realizó el trabajo \\ $\square$ Descriptores en español e inglés \\ $\square$ Abreviaturas \\ $\square$ Dirección, teléfono y número de fax del autor a quien se debe dirigir toda correspondencia \\ $\square$ Fuentes de apoyo y manifestación de conflictos de interés}

\section{Artículo (a doble espacio)}

$\square$ Carta de aprobación del estudio por Comité Ético - científico

$\square$ Resumen estructurado en español

$\square$ Resumen estructurado en inglés

$\square$ Introducción

$\square$ Materiales y métodos

$\square$ Resultados

Discusión

$\square$ Agradecimientos y colaboradores

$\square$ Referencias

$\square$ Cuadros, leyendas de las figuras, figuras

$\square$ Permiso para reproducir todo material previamente publicado y autorización de pacientes para publicar fotografías

$\square$ Carta de cada autor de asignación de derechos, responsabilidad de participación y declaración de financiamiento, si existiera (puede ser una, firmada por todos los autores).

La Revista Acta Médica Costarricense les informa que para 2011 los médicos interesados en recibir el ejemplar impreso a su dirección geográfica o apartado postal, deben solicitarlo por escrito a: actamedica@medicos.cr, anotando los siguientes datos:

Nombre completo:

Código:

Teléfono de habitación:

Dirección geográfica o apartado: 